\title{
Pulmonary arteriovenous fistula
}

\author{
Mersedeh Karvandi", Saeed Ranjbar \\ Taleghani Hospital, ShahidBeheshti University of Medical Sciences, Tehran, Iran \\ Email address: \\ mersedeh_karvandi@ipm.ir (M. Karvandi)
}

\section{To cite this article:}

Mersedeh Karvandi, Saeed Ranjbar. Pulmonary Arteriovenous Fistula. International Journal of Medical Imaging. Vol. 2, No. 2, 2014, pp. 34-38. doi: 10.11648/j.ijmi.20140202.16

\begin{abstract}
Pulmonary arteriovenous fistulas (PAVFs) are rare vascular malformations (PAVMs) of the lung that could lead to severe hypoxiemia due to right-to-left intrapulmonary shunts. They may occur as isolated entities or associated with Osler-Rendu-Weber syndrome or hereditary haemorrhagic telangiectasia (HHT). We study 3 patients with Rendu-OslerWeber disease and a large arteriovenous malformation involving the left pulmonary artery. We describe the successful checking pulmonary arteriovenous fistula using echocardiographic speckle tracking and velocity vector imaging to rule out sources of cardiac shunts.
\end{abstract}

Keywords: Right Upper Pulmonary Artery, Left Pulmonary Artery, Fistula, Contrast Echocardiography

\section{Introduction}

Abnormal development of the pulmonary arteries and veins in a common vascular complex is responsible for this congenital anomaly.Symptoms caused by pulmonary arteriovenousfistula (AVF) are often insidious, as the arteriovenousmalfunctions slowly enlarge. These abnormal communications have been given various names including pulmonary arteriovenous fistulae, pulmonary arteriovenous aneurysms, hemangiomas of the lung, cavernousangiomas of the lung, pulmonary telangiectases, and pulmonary arteriovenous malformations. The amount of right to left shunting depends on the extent of fistulous communication and may result cyanosis. Systolic and continuous murmurs may be audible over area of the fistula. Less common complaints include chest pain, cough, migraine headaches, tinnitus, dizziness, dysarthria, syncope, vertigo, and diplopia. The cause of these symptoms is not entirely clear, but it may be related to hypoxemia, polycythemia, or paradoxical embolization through the pulmonary arteriovenous malformations. Contrast echocardiography is helpful in the initial diagnostic process with the use of a saline contrast injection into a systemic vein. With pulmonary arteriovenous malformation, there is early pulmonary venous return to the left atrium, but as quickly as for patient with a PFO or ASD and right to left atrial shunting ${ }^{1-4}$.

We present images obtained from 3 patients with fistulous communication between left pulmonary artery (LPA) and right upper pulmonary veins (RUPV) referred for routine 2DTTE, 2DTEE, 2D and 4D strain imaging using echocardiographic speckle tracking and velocity vector imaging to rule out sources of cardiac shunts. Agitated saline contrast was performed during 2DTEE(Figures 1, 2, 3, 4, 5,6,7 and 8). 


\section{Method}

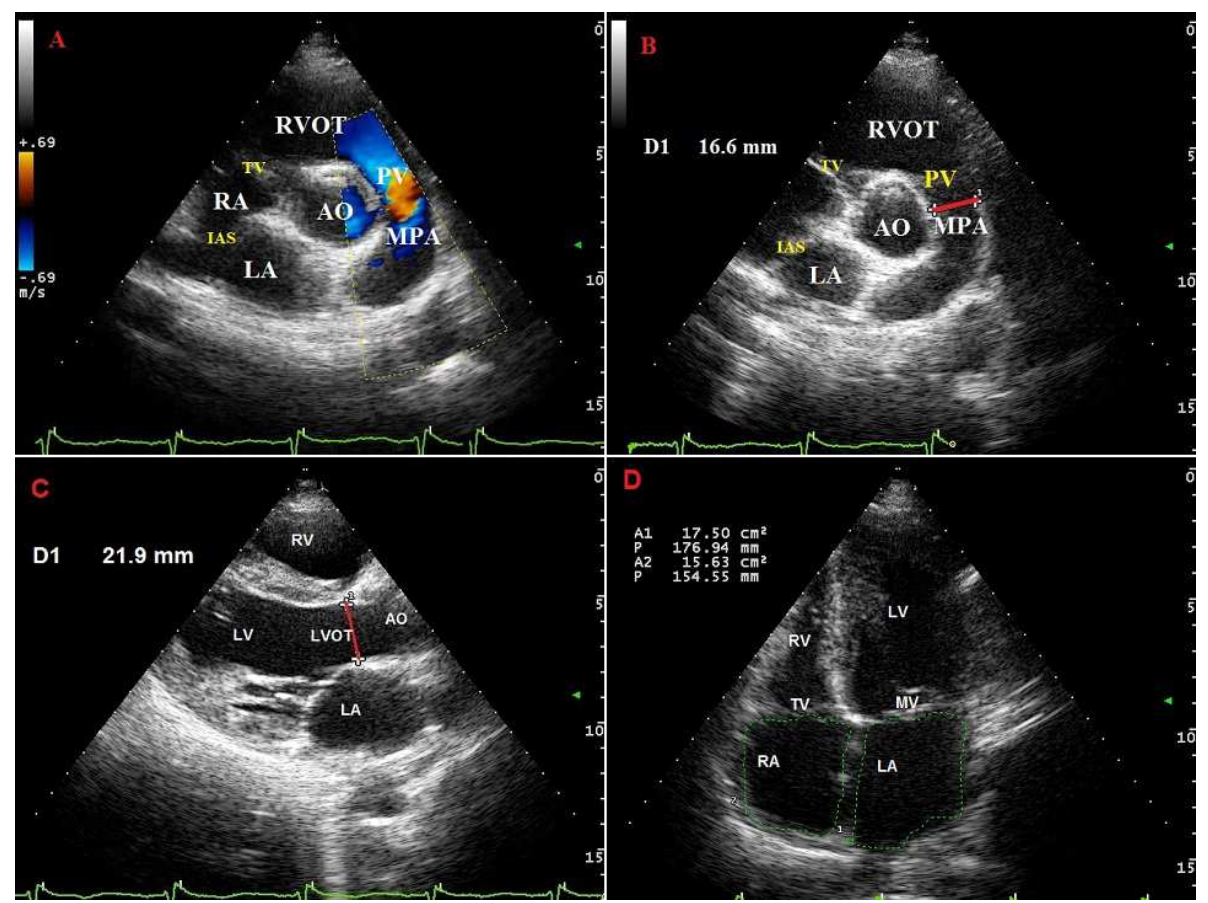

Figure 1.Parasternal short axis views at the level of the aortic valve are demonstrated for 3 patients (A to B). This is showing AO, aortic valve;RA, right atrium; LA, left atrium; RVOT,right ventricular outflow tract; PV, pulmonic valve; MPA, main pulmonary artery; IAS, inter-atrial septum; TV, tricuspid valve. Aortic valve is normal and is in open position(systolic frame) (A). Main pulmonary artery (MPA) size is 16.6 mm that is normal and pulmonary valve is also normal (B). Echocardiogram in parasternal long axis view showing left ventricular out flow track (LVOT) size $21.9 \mathrm{~mm}$ that is normal (C). Apical 4 chamber (4C) view with normal mitral valve (MV) and normal tricuspid valve (TV) and with normal left atrium (LA) and right atrium (RA) area sizes $17.50 \mathrm{~m}^{2}, 15.63 \mathrm{~m}^{2}$ respectively. $4 \mathrm{C}$ demonstrated also normal left ventricle (LV) size and its contractility and normal right ventricle (RV) size and its function (D).
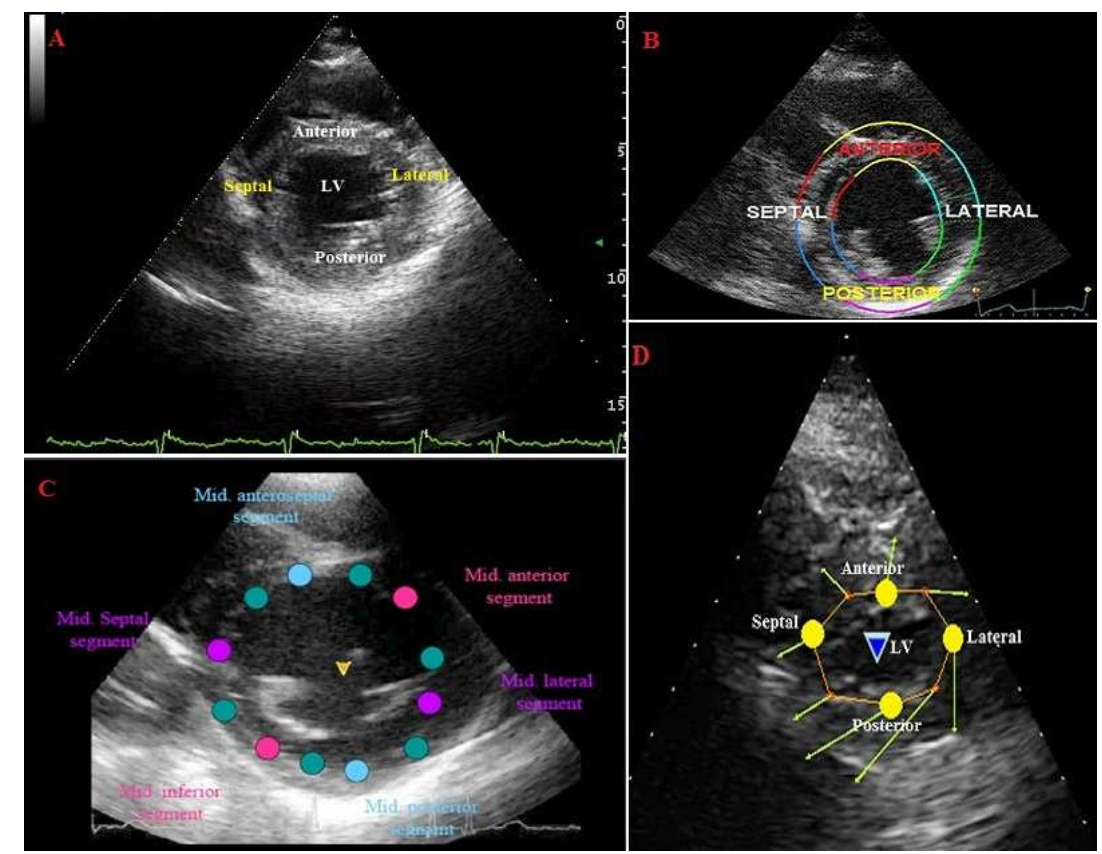

Figure 2.Parasternal short axis views at the level of the papillary muscle are established for 3 patients (A to D). Anterior, Posterior, Septal and Lateral walls have been determined. It showed left ventricular contractility is normal (A). In order to obtain strain and strain rate values for six segments (basal, mediumand "apical" lateral wall, and basal, medium, "apical" septum) we tracked13 points and picked up values of the paired ones(B). Region of interested is tracked by different colors to calculate radial and circumferential strains (C). Delineation of the apical myocardial short-axis view during vector velocity imaging Analysis and Accordingly, at the end of diastole, 12 and 8 tracking points were positioned at the mid-wall of thebasal and apical levels respectively. The system then applied a sequence of processing steps to track the motion of the segments frame to frame. The rotation of the left ventricle (LV) segments around the LV central axis at each shortaxis level was calculated separately on the basis of the average motion of the mid-wall points (D) 

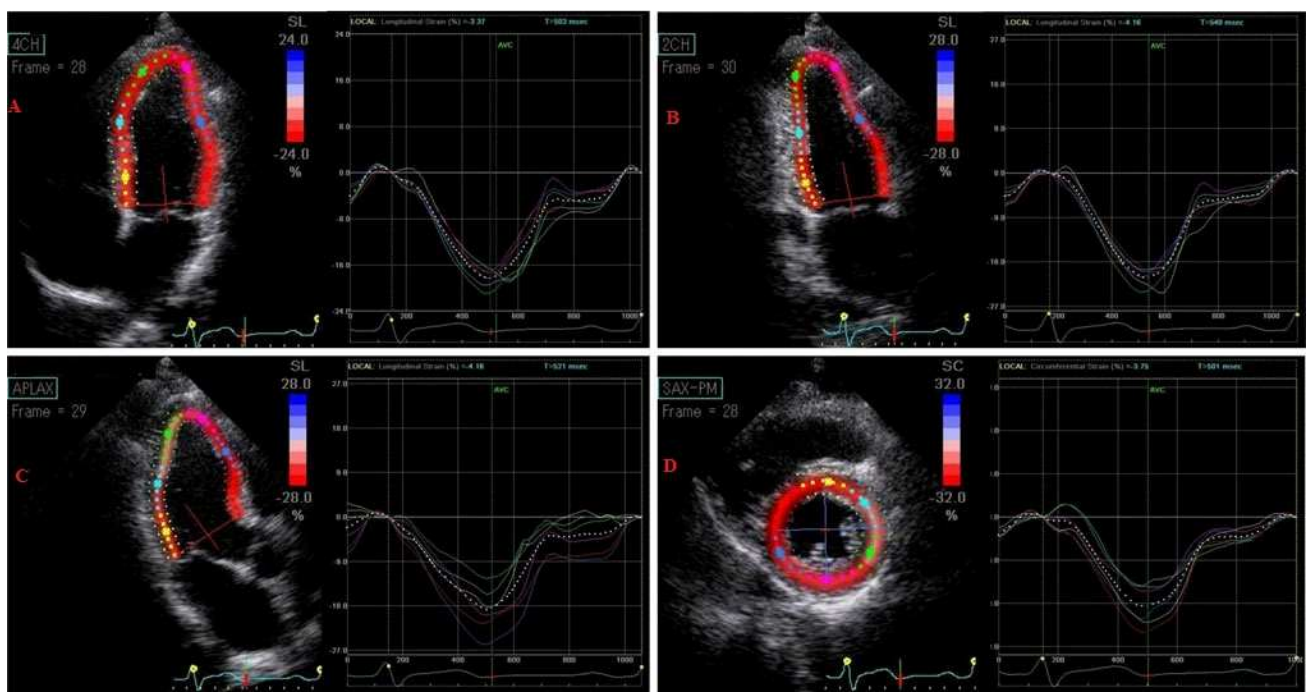

Figure 3.Speckle trackingExamples of global longitudinal strain measures from 3 standard apical views and global circumferential strain measures from the papillary muscle level in the parasternal short-axis view, The images obtained in the 4-chamber (A), 2-chamber (B),apical long-axis (C), and shortaxis (D)are shown. The color curves indicate color-coded segmental strain and dashedline indicate global strain.

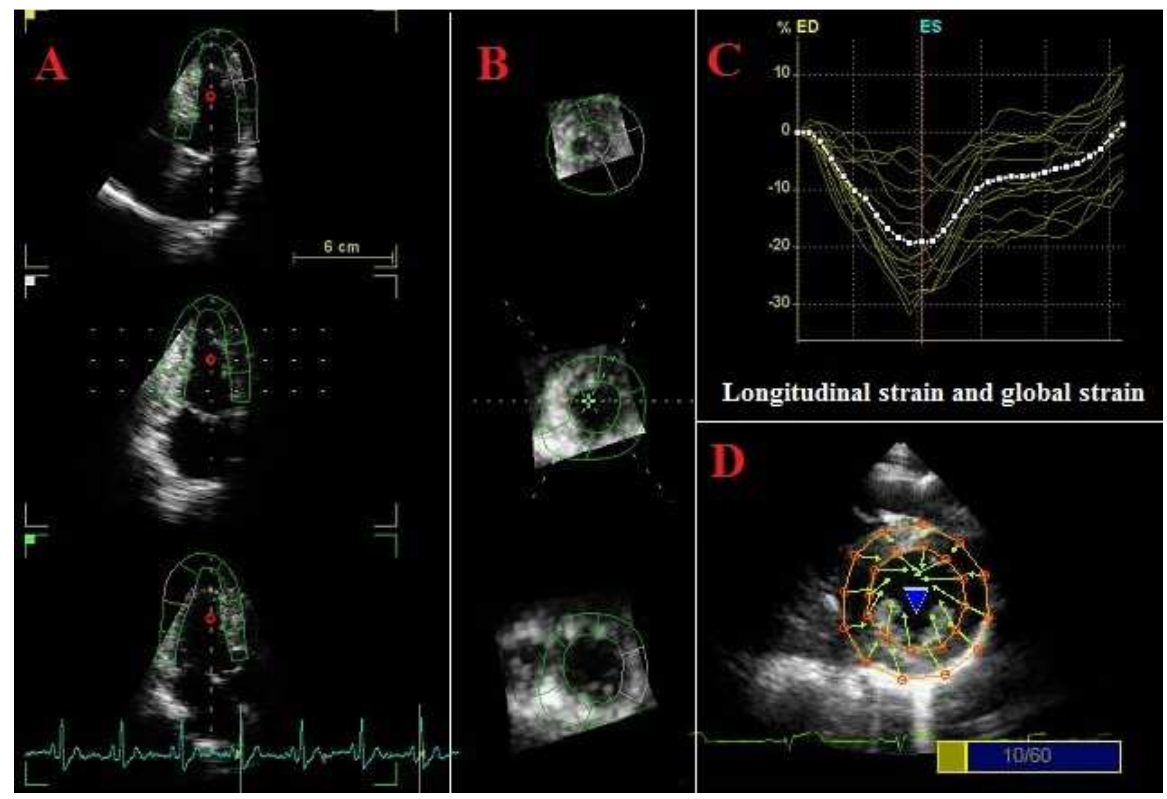

Figure 4.4D Strain results, longitudinal strainfor three patientsare presented (A to C). The $4 D$ Strain region of interest (ROI) shown in an alternative layout with a large 2-chamber slice and three SAX slices through the 4D dataset. This display allows the user to see if the ROI is placed correctly and also to easily assess the tracking quality (A to B). 4D Strain results from hearts of three patients, showing longitudinal strain and a plot with yellow traces for each of the 17 echocardiographic segments, in addition to a white trace for the global strain (C). Velocity vector imaging's have been applied in transthoracic electrocardiogram short axis views at the level of the papillary muscles: endocardial and epicardial borders with arrows showing the local directions of border motions that for our cases theses vector directions are normal. Velocity for all points on borders as functions of time, and displayed as a tissue Doppler imaging-style color map (D)

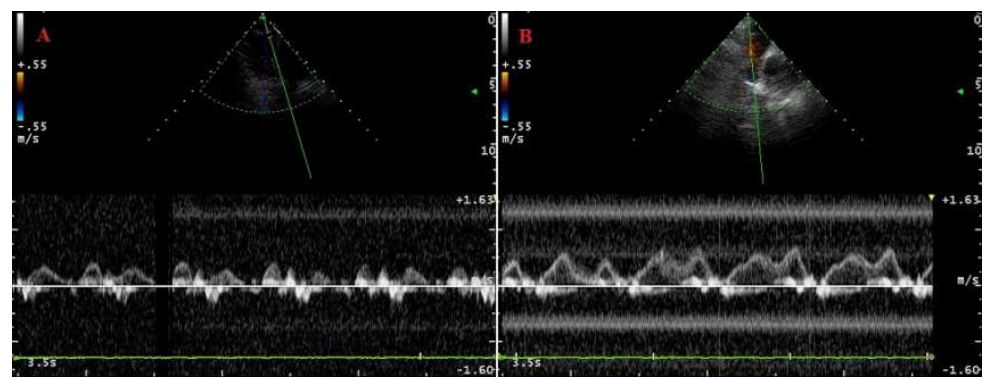

Figure 5.TEE in plane $110^{\circ}$ view showed normal flow in left pulmonary veins (A). TEE in plane $50^{\circ}$ view demonstrated normal flow of right upper pulmonary veins (no abnormal drain) (B) 


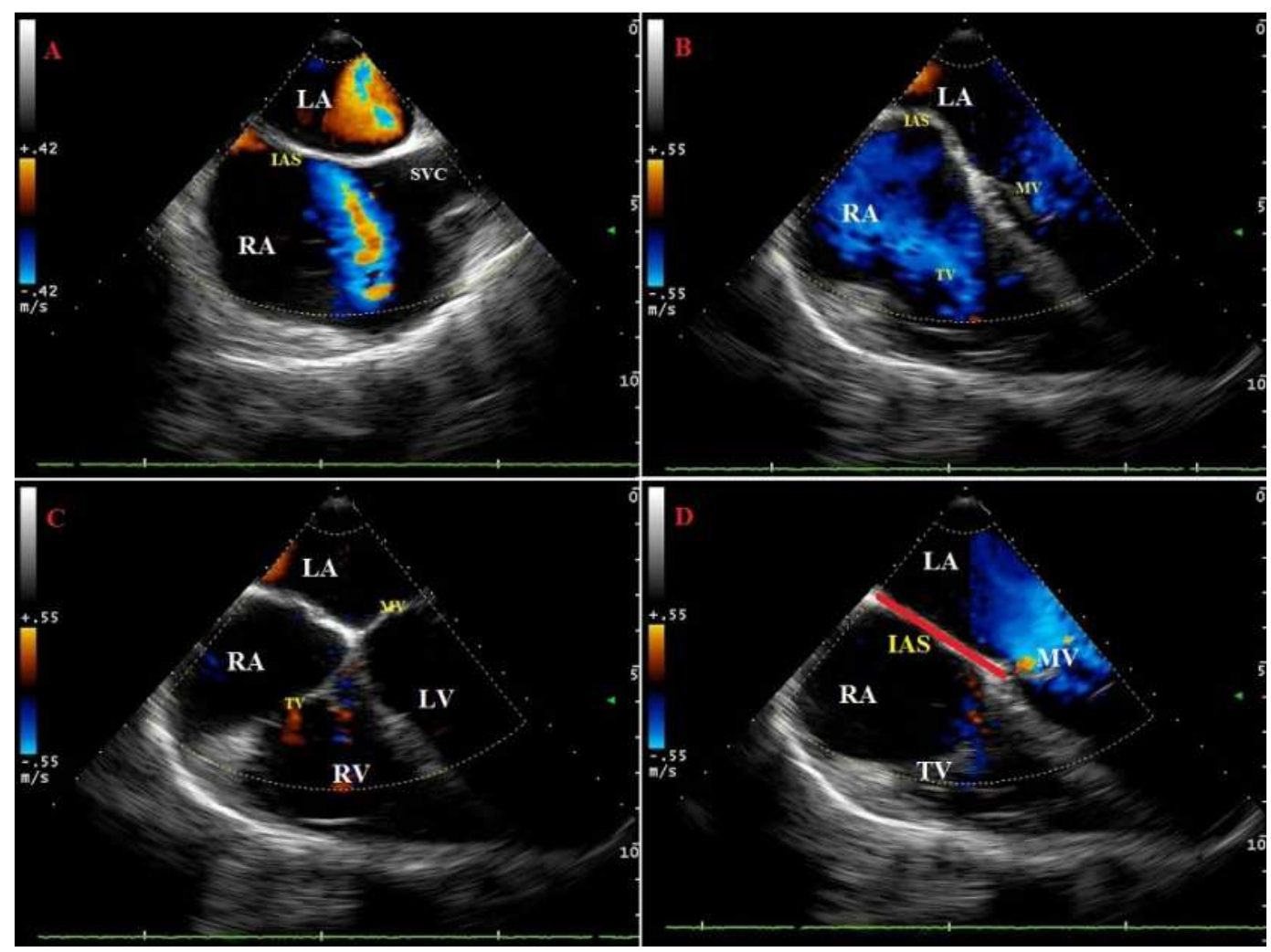

Figure 6.Modifiedcaval view shows normal inter-atrial septum. RA indicates right atrium; LA, left atrium; IAS, inter-atrial septum; SVC, superior vena cava (A). TEE views confirm also normal mitral valve and normal tricuspid valve. The normal inter-atrial septum illustrates that there is no right to left atrial shunting (ASD). MV, mitral valve; TV, tricuspid valve; $A S D$, atrial septal disorder (B to D)
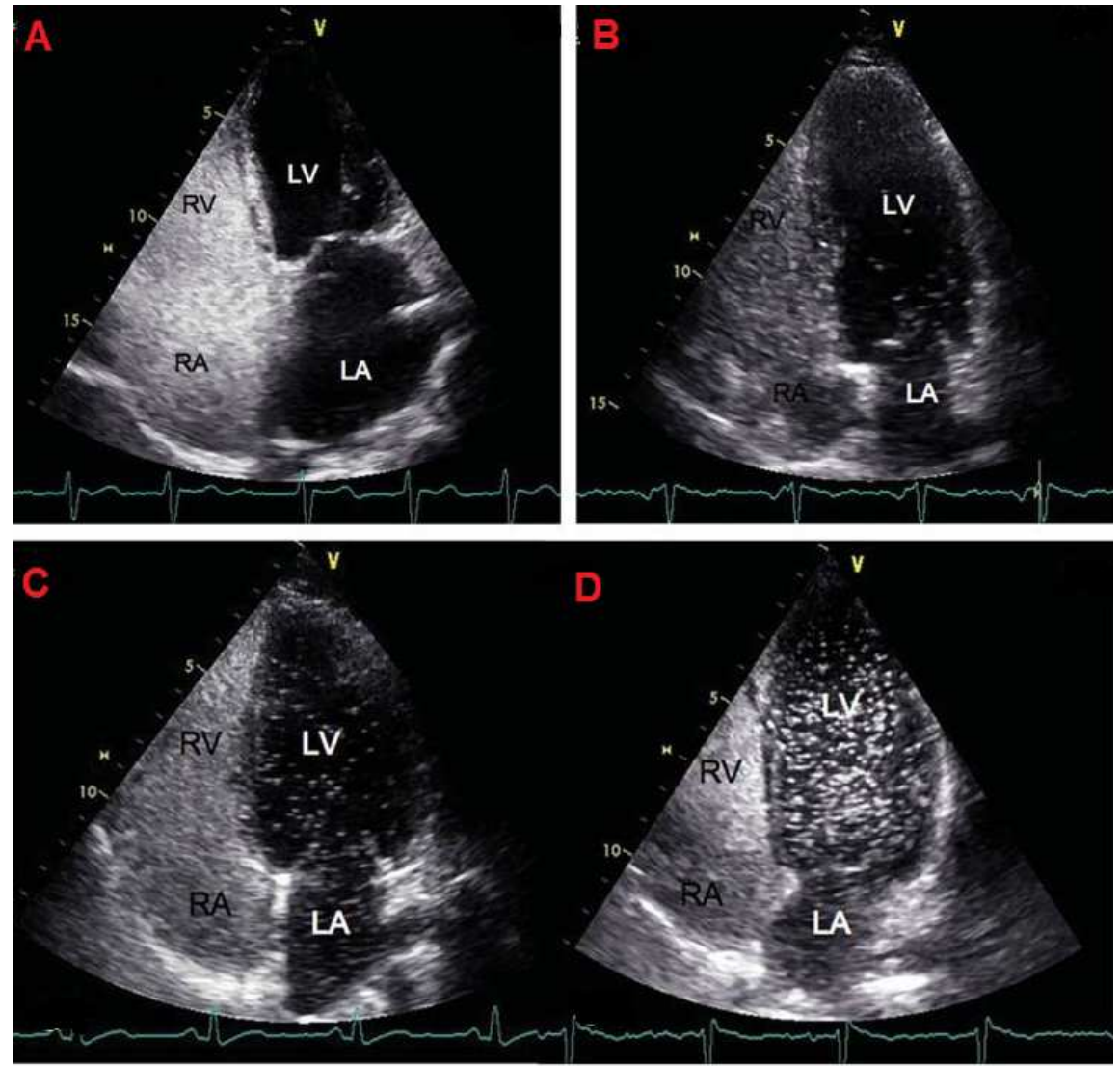

Figure 7. With contrast echocardiography, after iv injection of agitated saline solution, left ventricular opacification was graded as: (A) Grade 0, no bubbles; (B) 1, occasional filling with,20 bubbles; (C) 2, moderate filling, with discrete bubbles, either they are less or more numerous (C to D). 


\section{Result}

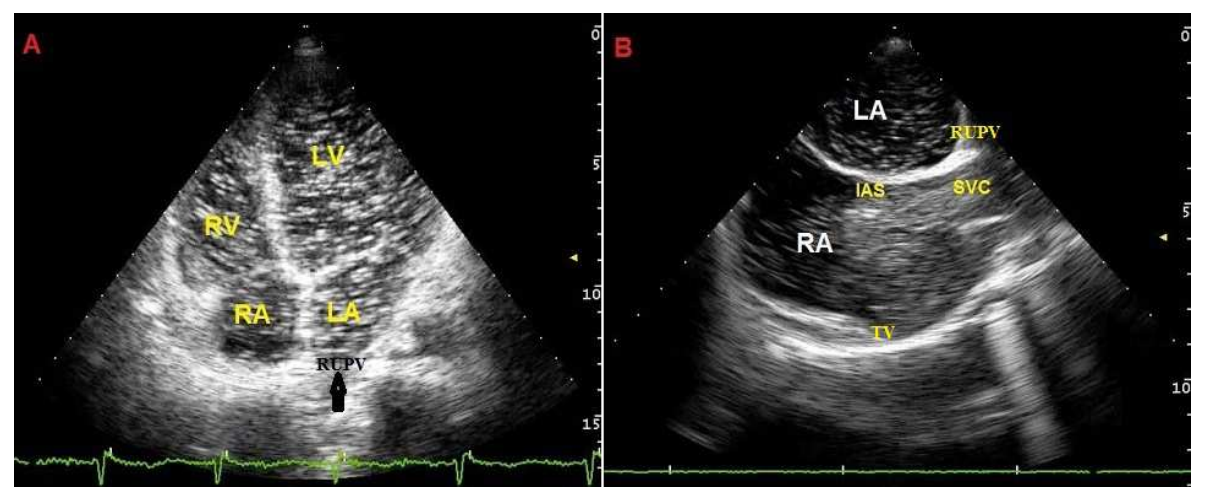

Figure 8.Modified cavalview and contrast injection: a complete visualization of right ventricle (RV), right atrium (RA), left ventricle (LV), left atrium (LA) are cleared. Echocardiogram views showing fistulous communication between left pulmonary artery (LPA) and right upper pulmonary veins (RUPV). Bubble contrast echocardiogram demonstrating filling of left atrium from the right upper pulmonary veins (thickening arrow)(A to B) and (Videos 1 and 2). TV indicates tricuspid valve.

\section{Conclusion}

Novel echocardiographic technologies provide high quality images of functions and structures of the heart to diagnose of cardiac diseases.

\section{Disclosure}

There is no conflict of interest.

\section{References}

[1] Jmaes R. Gossage K. Ghassan K. Pulmonary Arteriovenous Malformations.Am J RespirCrit Care Med. 1998: 158: 643661.

[2] Remy J, Remy-Jardin M, Giraud F, Wattinne L. Angioarchitecture of pulmonary arteriovenous malformations: clinical utility of three dimensional helical CT. Radiology 1994;191:657-64.

[3] Gossage JR, Kanj G. Pulmonary arteriovenous malformations. A state of the art review. Am J RespirCrit Care Med. 1998:158: 43-61.

[4] Gupta P, Mordin C, Curtis J, Hughes JM, Shovlin CL, Jackson JE. Pulmonary arteriovenous malformations: effect of embolization on right to left shunt, hypoxemia, and exercise tolerance in 66 patients. AJR Am J Roentgenol. 2002:179: 47-55. 